\title{
Influencia de la exposición gingival en la percepción estética de la sonrisa.
}

\author{
Gingival influence of exposure in the perception of aesthetic smile. \\ Rosella Flores-Vignolo ${ }^{l, a}$, Abraham Meneses-López ${ }^{2, b}$, Carlos Liñán-Durán ${ }^{1, b}$
}

\section{RESUMEN}

Objetivo: Evaluar la influencia de los diferentes niveles de exposición gingival sobre la percepción estética de la sonrisa en pacientes y residentes del postgrado de Ortodoncia de la Universidad Peruana Cayetano Heredia (UPCH). Material y métodos: Diseño de corte observacional, descriptivo, transversal y comparativo. La muestra estuvo conformada por 36 pacientes y 35 residentes del postgrado de Ortodoncia de la UPCH. Para la ejecución del estudio se utilizó una fotografía del rostro durante la sonrisa de una mujer, la cual fue modificada por un especialista en computación mediante el programa Adobe Photoshop en 5 niveles diferentes de exposición gingival: $-2 \mathrm{~mm}, 0 \mathrm{~mm},+2 \mathrm{~mm},+4 \mathrm{~mm}$ y $+6 \mathrm{~mm}$. La percepción de los dos grupos de evaluadores fue determinada mediante una escala visual análoga (EVA). Resultados: Al evaluar la influencia del grupo de estudio en la percepción de la sonrisa, no se encontraron diferencias estadísticamente significativas entre pacientes y residentes $(\mathrm{p}=0.096$ ). Conclusiones: Las exposiciones gingivales de $0 \mathrm{~mm}$ y $-2 \mathrm{~mm}$ recibieron los calificativos más altos por ambos grupos.

Palabras clave:PERCEPCIÓN/SONRISA/GINGIVAL.

\begin{abstract}
Objective: To evaluate the influence of different levels of gingival exposure on smile aesthetics perception in patients and postgraduate orthodontic residents of Peruvian University Cayetano Heredia (UPCH). Materials and Methods: Study design cut observational, descriptive, cross- sectional and comparative. Sample consisted of 36 patients and 35 orthodontic residents of UPCH. To carry out the study was used a photography of the face during the smile of a woman, which was modified by a computer specialist by the Adobe Photoshop program in 5 different levels of gingival exposure: -2mm, Omm, $+2 \mathrm{~mm},+4 \mathrm{~mm}$ and $+6 \mathrm{~mm}$. The perception of the two groups of raters was determined by a visual analog scale (VAS). Results: In assessing the influence of the study group's perception of the smile, no statistically significant difference between patients and residents ( $p=0.096)$ were found. Conclusions: Gingival exposures 0mm and -2mm received the highest scores for both groups.
\end{abstract}

Key words: PERCEPTION/SMILE / GINGIVAL.

\footnotetext{
Facultad de Estomatología. Universidad Peruana Cayetano Heredia. Lima Perú.

2 Sociedad Peruana de Ortodoncia. Lima Perú.

a Residente de la Especialidad de Ortodoncia.

b Especialista en Ortodoncia.
} 


\section{INTRODUCCIÓN}

En las últimas décadas, la demanda por los servicios cosméticos se ha incrementado considerablemente en muchas partes del mundo. Varias especialidades médicas proporcionan servicios cosméticos siendo testigos del incremento de procedimientos que realzan los rasgos físicos, revirtiendo el paso de los años y mejorando la estética. Los procedimientos cosméticos quirúrgicos, el relleno de la piel, procedimientos ortodoncicos y ortognáticos, el blanqueamiento dental y otros procedimientos dentales cosméticos son ampliamente requeridos por los adultos (1).

La estética se determina como el reflejo de una apariencia agradable. Peck y Peck (2) definieron el concepto de "estético" como la apreciación de verse bien o percepción de la belleza. Esta identificación está relacionada a una sensación agradable sobre la exposición de un objeto, un sonido o una persona. Por lo tanto, el concepto de belleza es único para cada individuo, y es establecido basándose en valores como el género, raza, educación y experiencias personales. La comparación entre los estándares individuales a través del tiempo es debido a la globalización del concepto de belleza (3).

Tener una sonrisa hermosa y juvenil es el principal consenso entre los pacientes. Las mejorías estéticas son requeridas frecuentemente en los consultorios dentales. En este contexto, la cantidad de exposición gingival es fundamentalmente importante para una sonrisa agradable, pues la mayoría de personas consideran una sonrisa gingival antiestética (4).

La capacidad de mostrar encía al sonreír depende de varios factores. Así, la solución de este problema para conseguir niveles óptimos de exposición gingival es usualmente difícil porque requiere la identificación y corrección exacta de la causa del problema, que puede ser esquelético, dental o ambos(5).

Kokich y Col. (6) evaluaron también el criterio estético, entre ellos la percepción de la cantidad de la exposición gingival, usando fotografías de sonrisa que fueron modificadas intencionalmente con una computadora. Las variaciones entre la distancia del labio superior hasta los incisivos superiores (margen gingival) fue introducido, generando 5 tipos de imágenes: $2 \mathrm{~mm}$ de los incisivos cubiertos por los labios, los labios tocando el margen gingival de los incisivos (0mm de exposición gingival) y $2 \mathrm{~mm}, 4$ $\mathrm{mm}$ y $6 \mathrm{~mm}$ de exposición gingival. Las imágenes fueron evaluadas por ortodoncistas, pacientes y dentistas generales. Los resultados demostraron que la exposición gingival mayor de $4 \mathrm{~mm}$ fue considerada aceptable por los últimos dos grupos de individuos, pero los ortodoncistas consideraron la exposición mayor de $2 \mathrm{~mm}$ antiestética.

Hunt y Col. (7) y Geron (8) usaron imágenes manipuladas con variaciones en la cantidad de exposición gingival al sonreír para evaluar la percepción estética de la sonrisa gingival. Estos autores solo evaluaron la percepción de los pacientes y no hicieron comparación con otro grupo de examinadores. De acuerdo con Hunt y Col. (7) las sonrisas con más de $2 \mathrm{~mm}$ de exposición gingival fueron consideradas antiestéticas, y la sonrisa ideal fue la que no presentaba exposición gingival. La literatura también sugiere que las mujeres son más tolerantes de las variaciones en estas características que los hombres (8).

El propósito del estudio fue comparar la percepción estética de los pacientes asistentes a la clínica dental docente-asistencial de la Universidad Peruana Cayetano Heredia con la percepción de estudiantes de post grado de ortodoncia de la Facultad de Estomatología de la Universidad Peruana Cayetano Heredia.

\section{MATERIALES Y MÉTODOS}

El diseño del estudio fue observacional, descriptivo, transversal y comparativo. La muestra estuvo conformada por 36 pacientes y 35 residentes del postgrado de Ortodoncia de la Universidad Peruana Cayetano Heredia. Se utilizó la fotografía de una mujer, quien previamente fue notificada sobre el propósito del estudio y una vez que aceptó participar en el estudio voluntariamente, firmó un consentimiento informado. Se le tomó una fotografía utilizando una cámara Nikon D3100 apoyada sobre un trípode, a una distancia de $30 \mathrm{~cm}$ de una pared de fondo blanco y a $1 \mathrm{~m}$ de la cámara con el rostro mirando al frente durante la sonrisa y el plano de Frankfort paralelo al piso. El rostro de la fotografía evaluada, fue elegido por un juicio de expertos, conformado por docentes del post grado de Ortodoncia de la Facultad de Estomatología 
de la Universidad Peruana Cayetano Heredia, quienes determinaron una sonrisa estéticamente aceptable, mediante una encuesta donde se les mostró las fotografías de los rostros elegidos y ellos indicaron si estaban de acuerdo o no con el nivel de exposición gingival natural mostrado. Luego de tener la fotografía elegida por los expertos, esta fue modificada por un técnico en computación mediante el programa Adobe Photoshop para obtener 5 variaciones en el nivel de exposición gingival $(-2 \mathrm{~mm}, 0 \mathrm{~mm},+2 \mathrm{~mm},+4 \mathrm{~mm}$, $+6 \mathrm{~mm}$ ) para cada fotografía (Fig. 1). De este modo se obtuvieron cinco fotografías con diferentes niveles en la exposición gingival. A cada participante se le explicó el propósito del estudio y se le preguntó a cada uno de ellos si aceptaban participar voluntariamente en la investigación. Se les indicó que visualizarían el grupo de fotografías por un período máximo de 30 segundos, transcurrido este tiempo tendrían que marcar su respuesta, además se les advirtió que no podrían reevaluar las fotografías previamente vistas. La encuesta constó de una pregunta con alternativas múltiples y una escala visual analóga (EVA) para calificar el nivel de agrado de la fotografía escogida. Una vez que se recolectaron todas las encuestas, los resultados fueron digitados y codificados en una hoja de cálculo para su posterior análisis.

\section{RESULTADOS}

Se encuestó a 71 personas $(30$ hombres y 41 mujeres), quienes aceptaron evaluar voluntariamente un grupo de fotografías (pertenecientes al rosto de una mujer). El grupo de fotografías incluía cinco fotografías de la misma persona en máxima sonrisa mostrando diferentes niveles de exposición gingival: $-2 \mathrm{~mm}, 0 \mathrm{~mm}$, $2 \mathrm{~mm}, 4 \mathrm{~mm}$ y $6 \mathrm{~mm}$ respectivamente. Los presentes resultados se basaron en las respuestas de las personas entrevistadas que correspondieron a 36 pacientes y 35 residentes (tabla 1 y 2 ).

Utilizamos la prueba Kolmogorov-Smirnov para valorar nuestra muestra, que tenía una distribución normal. Por ello, posteriormente usamos la prueba de $\mathrm{T}$ student para comprar los dos grupos entrevistados en el presente estudio.

Al evaluar la influencia del grupo de estudio en la percepción de la sonrisa (tabla 3), no se encontraron diferencias estadísticamente significativas entre pacientes y residentes $(p=0.096)$. La sonrisa más seleccionada por pacientes y residentes de ortodoncia fue la que no presentaba exposición gingival, $0 \mathrm{~mm}$. El grupo de residentes fue el que eligió por más veces la fotografía que no mostraba exposición gingival. $(33.33 \%-60.00 \%)$.

\section{DISCUSIÓN}

En el presente estudio se buscó obtener información con respecto a la percepción del atractivo dental, particularmente sobre la influencia que ejerce la exposición gingival en la percepción de la sonrisa en nuestra población, mediante encuestas.

Se utilizó para realizar las encuestas la escala visual análoga (EVA) puesto que ya ha sido aplicada para evaluar la intensidad del dolor y se ha demostrado que es un método válido, fiable y reproducible para medir el dolor subjetivo.9 Como muchos investigadores, 10-15 también nos valimos del método para juzgar el atractivo de la sonrisa, pues en la estética también proporciona resultados sencillos, rápidos y reproducibles. Además para la construcción del instrumento de encuesta, se utilizó el método de modificación de fotografías mediante el programa

Tabla 1. Comparación de la percepción de la sonrisa por sexo del evaluador

\begin{tabular}{|c|c|c|c|c|c|c|}
\hline \multirow{3}{*}{ Percepción de la Sonrisa } & \multicolumn{4}{|c|}{ Sexo } & & \\
\hline & \multicolumn{2}{|c|}{ Masculino } & \multicolumn{2}{|c|}{ Femenino } & \multicolumn{2}{|c|}{ Total } \\
\hline & $\mathrm{n}$ & $\%$ & $\mathrm{n}$ & $\%$ & $\mathrm{n}$ & $\%$ \\
\hline Menos $2 \mathrm{~mm}$ de exposición & 8 & 26.67 & 14 & 34.15 & 22 & 30.99 \\
\hline 0mm de exposición & 14 & 46.67 & 19 & 46.34 & 33 & 46.48 \\
\hline $2 \mathrm{~mm}$ de exposición & 5 & 16.67 & 6 & 14.63 & 11 & 15.49 \\
\hline $4 \mathrm{~mm}$ de exposición & 2 & 6.57 & 1 & 2.44 & 3 & 4.23 \\
\hline 6mm de exposición & 1 & 3.33 & 1 & 2.44 & 2 & 2.82 \\
\hline
\end{tabular}


Tabla 2. Comparación de la percepción de la sonrisa por tipo de evaluador

\begin{tabular}{|c|c|c|c|c|c|c|}
\hline \multirow{3}{*}{ Percepción de la Sonrisa } & \multicolumn{4}{|c|}{ Grado de Instrucción } & & \\
\hline & \multicolumn{2}{|c|}{ Paciente } & \multicolumn{2}{|c|}{ Residente } & \multicolumn{2}{|c|}{ Total } \\
\hline & $\mathrm{n}$ & $\%$ & $\mathrm{n}$ & $\%$ & $\mathrm{n}$ & $\%$ \\
\hline Menos $2 \mathrm{~mm}$ de exposición & 10 & 27.78 & 12 & 34.29 & 22 & 30.99 \\
\hline 0mm de exposición & 12 & 33.33 & 21 & 60.00 & 33 & 46.48 \\
\hline $2 \mathrm{~mm}$ de exposición & 10 & 27.78 & 1 & 2.86 & 11 & 15.49 \\
\hline 4mm de exposición & 3 & 8.33 & 0 & 0.00 & 3 & 4.23 \\
\hline $6 \mathrm{~mm}$ de exposición & 1 & 2.78 & 1 & 2.86 & 2 & 2.82 \\
\hline Total & 36 & 50.70 & 35 & 49.30 & 71 & 100.00 \\
\hline
\end{tabular}

Adobe PhotoShop como en varios estudios similares realizados con anterioridad. 6 - 8, $16-17$

Se comparó el atractivo de la exposición gingival mediante cinco niveles diferentes, los cuales variaban desde $2 \mathrm{~mm}$ de cobertura del labio superior a los incisivos centrales superiores, hasta $6 \mathrm{~mm}$ de exposición gingival. En estudios anteriores que evaluaron la influencia de la exposición gingival en el atractivo de una sonrisa, las imágenes fueron modificadas de $-2 \mathrm{~mm}$ a $4 \mathrm{~mm}$, 7 ó de -4.6 a $3.3 \mathrm{~mm}$, 8 de exposición gingival. La gama evaluada en esta investigación fue lo suficientemente amplia para la comparación con los estudios anteriores.

Al evaluar la muestra de los examinadores residentes de ortodoncia prefirieron a la fotografía que no mostraba exposición gingival, $0 \mathrm{~mm}, \mathrm{y}$ aquella que presentaba cubrimiento del labio superior a los incisivos centrales superiores, $-2 \mathrm{~mm},(60.00 \%$ - 34.28\%), respectivamente. Este resultado es similar con los resultados de Peck y Peck, 2 quienes afirmaron que las variaciones de hasta $1 \mathrm{~mm}$ de exposición gingival se consideran estéticamente agradables para todos los grupos. Este resultado también sugiere que, en la sonrisa ideal, no hay exposición gingival y/o el labio superior descansa muy cerca del margen gingival de los incisivos centrales superiores.4, 7, 8

Kokich et al, 6 encontraron que los pacientes consideraban que la exposición gingival hasta $4 \mathrm{~mm}$ era aceptable, mientras los ortodoncistas consideraban que la exposición gingival de más de 2 $\mathrm{mm}$ era antiestética. Estos resultados coinciden con los del presente estudio, ya que el $8.33 \%$ del grupo de pacientes eligió una sonrisa con $4 \mathrm{~mm}$ de exposición gingival contrastando con el $0.00 \%$ de elección a esta misma fotografía por parte del grupo de residentes. Además coincide también los resultados para el grupo de residentes, quienes tuvieron preferencia por la fotografía que no mostraba exposición gingival, $0 \mathrm{~mm}, 60.00 \%$.

Según el informe de Ioi et al, 18 los resultados de este estudio son semejantes con lo que ellos obtuvieron. La sonrisa sin exposición gingival, $0 \mathrm{~mm}$, se consideró la más atractiva por los ortodoncistas y la sonrisa con $2 \mathrm{~mm}$ de cobertura de labio superior sobre los incisivos centrales superiores fue considerada como la más atractiva por los estudiantes dentales. Por el grado de instrucción podríamos comparar el último grupo mencionado con el grupo de pacientes

Tabla 3. Comparación de la percepción de la sonrisa por tipo de evaluador/sexo

\begin{tabular}{|c|c|c|c|c|c|c|c|c|c|c|c|c|}
\hline \multicolumn{13}{|c|}{ Grado de Instrucción } \\
\hline \multirow{3}{*}{ Percepción de la Sonrisa } & \multicolumn{6}{|c|}{ Paciente } & \multicolumn{6}{|c|}{ Residente } \\
\hline & \multicolumn{2}{|c|}{ Femenino } & \multicolumn{2}{|c|}{ Masculino } & \multicolumn{2}{|c|}{ Total } & \multicolumn{2}{|c|}{ Femenino } & \multicolumn{2}{|c|}{ Masculino } & \multicolumn{2}{|c|}{ Total } \\
\hline & $\mathrm{n}$ & $\%$ & $\mathrm{n}$ & $\%$ & $\mathrm{n}$ & $\%$ & $\mathrm{n}$ & $\%$ & $\mathrm{n}$ & $\%$ & $\mathrm{n}$ & $\%$ \\
\hline Menos $2 \mathrm{~mm}$ de exposición & 8 & 34.78 & 2 & 15.38 & 10 & 27.78 & 6 & 33.33 & 6 & 35.29 & 12 & 34.29 \\
\hline 0mm de exposición & 9 & 39.13 & 3 & 23.08 & 12 & 33.33 & 10 & 55.56 & 11 & 64.71 & 21 & 60.00 \\
\hline $2 \mathrm{~mm}$ de exposición & 5 & 21.74 & 5 & 38.46 & 10 & 27.78 & 1 & 5.56 & 0 & 0.00 & 1 & 2.86 \\
\hline $4 \mathrm{~mm}$ de exposición & 1 & 4.35 & 2 & 15.38 & 3 & 8.33 & 0 & 0.00 & 0 & 0.00 & 0 & 0.00 \\
\hline 6mm de exposición & 0 & 0 & 1 & 7.69 & 1 & 2.78 & 1 & 5.56 & 0 & 0.00 & 1 & 2.86 \\
\hline Total & 23 & 63.89 & 13 & 36.11 & 36 & 100.0 & 18 & 51.43 & 17 & 48.57 & 35 & 100.00 \\
\hline
\end{tabular}




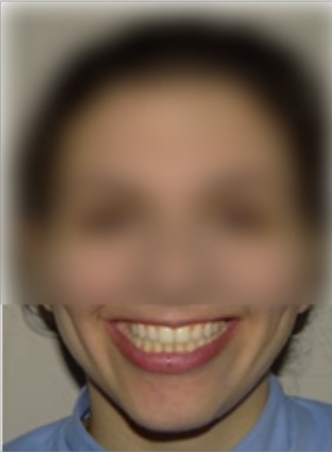

$-2 \mathrm{~mm}$

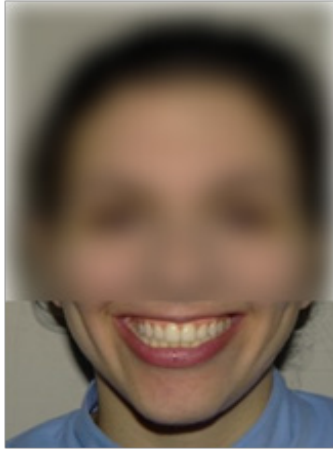

$0 \mathrm{~mm}$

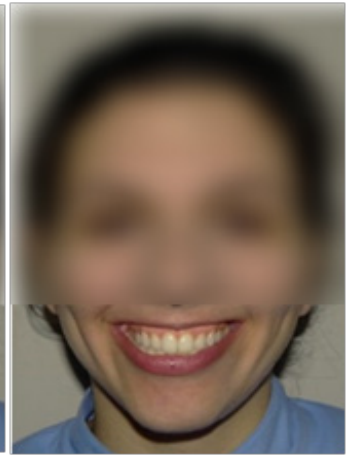

$2 \mathrm{~mm}$

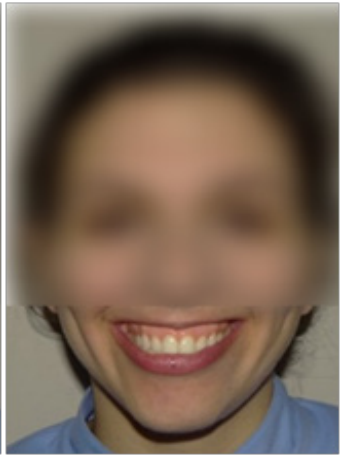

$4 \mathrm{~mm}$

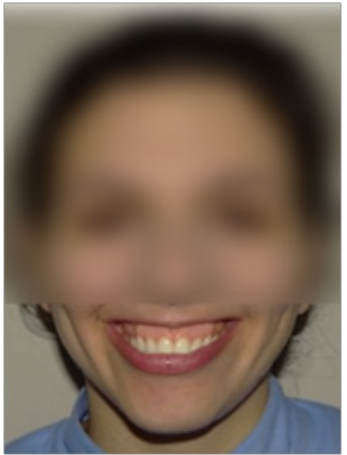

$6 \mathrm{~mm}$ del presente estudio, quienes tuvieron preferencia al igual que los residentes por la fotografía sin exposición gingival; Sin embargo como segunda opción tuvieron preferencia por la fotografía con $2 \mathrm{~mm}$ de cobertura de labio superior sobre los incisivos centrales superiores $(33.33 \%-27.77 \%)$. Geron et al, 8 investigaron la percepción estética de la sonrisa en los pacientes, e informaron que la fotografía de sonrisa más atractiva para este grupo era la que no presentaba exposición gingival y la que presentaba cobertura del labio superior sobre los incisivos centrales alrededor de $2 \mathrm{~mm}$ respectivamente.

Los resultados encontrados en este estudio difieren a los de algunos autores, probablemente debido a los diferentes métodos y parámetros dento-faciales utilizados en la investigación. Por ejemplo, Kokich et al, 6 realizó un estudio similar, pero cambio el espesor y la forma del labio superior en sus imágenes, por lo tanto complicaba el análisis del efecto de la sonrisa gingival. Además, sabemos que el concepto de belleza no es absoluto sino subjetivo, a pesar de algunos parámetros predeterminados. Por lo tanto, la percepción estética de la sonrisa es algo muy personal y varía según la sensibilidad de cada sujeto. 3

Geron et al, 8 y Abu et al, 19 informaron que los evaluadores masculinos y femeninos puntuaron a las fotografías con exposición gingival de manera diferente, sugiriendo que los evaluadores femeninos son más tolerantes a la exposición gingival. Esto no ha sido corroborado con los resultados de nuestro estudio, pues los evaluadores masculinos tuvieron preferencia por la fotografía que no mostraba exposición gingival, $0 \mathrm{~mm}(46.66 \%)$ al igual que los evaluadores femeninos (46.34\%). Nuestros resultados coinciden con los de Moore et al, 20 e Ioi et al, 15 quienes informaron que los evaluadores masculinos $\mathrm{y}$ femeninos puntuaron las fotografías de una manera muy similar.

Varios especialistas de la odontología como ortodoncia $y$ en especial periodoncia, han manifestado recientemente una destacada tendencia a tratar a los pacientes con el objetivo de mejorar la estética de la sonrisa.21 Margolis, 22 señaló que los ortodoncistas como profesionales de la odontología están entrenados para creer que los pacientes deben mostrar la altura de los incisivos, más $2.1 \mathrm{~mm}$ de encía al sonreír. No obstante, aunque la literatura informa de algunas opiniones clínicas sobre el ideal o el nivel aceptable de exposición gingival, la mayoría de los especialistas no tienen ninguna base científica. Por lo tanto, la percepción estética de los pacientes, sobre todo en comparación con la de los ortodoncistas, debería ser importante y tomarse en cuenta al momento de la planificación de los tratamientos de ortodoncia.

Las limitaciones de este estudio incluyen el uso de una sonrisa femenina como la única imagen modelo pues se ha demostrado que el género de la imagen afecta la puntuación en el atractivo de una sonrisa.8 Además, aunque la muestra fue comparativa y similar a otros estudios, 7, 8, 11, 16, 23, 24 se aconseja si se realiza un estudio similar procurar homogenizar la cantidad de participantes tanto por género, edad y grado de instrucción para obtener valores más proporcionales y confiables. Por lo tanto se necesitan más estudios, con muestras más numerosas y con la introducción de nuevas variables como el grupo étnico del encuestado, razón socioeconómica, etc. Para explorar este tema, poco investigado en nuestro país pero de gran interés debido a la gran diversidad racial y cultural observada en el Perú. 


\section{CONCLUSIONES}

Las exposiciones gingivales de $0 \mathrm{~mm}$ y $-2 \mathrm{~mm}$ recibieron los calificativos más altos por ambos grupos de evaluadores respectivamente. Los pacientes dieron los puntajes más altos en comparación con los residentes.

Las exposiciones de $2 \mathrm{~mm}, 4 \mathrm{~mm}$ y $6 \mathrm{~mm}$ fueron percibidas de manera diferente por los distintos grupos. Los residentes fueron más estrictos en su puntuación en comparación con los pacientes.

\section{CORRESPONDENCIA}

Rosella Isabel Flores Vignolo

Av. Sánchez Carrión N²80 Dpto 206

Teléfono: 422-3286

e-mail: Rosella.flores@upch.pe

\section{REFERENCIAS BIBLIOGRÁFICAS}

1. Rohrich RJ. The increasing popularity of cosmetic surgery procedures: a look at statistics in plastic surgery. PlastReconstrSurg 2000;106(6):1363-5.

2. Peck H, Peck S. A concept of facial esthetics. Angle Orthod 1970;40(4):284-318.

3. Reis SAB, Abrão J, Capezolla LF, Claro CAA. Análise facial subjetiva. R Dental PressOrtodonOrtop Facial 2006;11(5):159-72.

4. Hulsey CM. An esthetics evaluation of lip-teeth relationships present in the smile. Am J Orthod 1970;57(2):132-44.

5. Ahmad I. Geometric consideration in anterior dental aesthetics: restorative principles. PractPeriodontic Aesthetic Dent 1998;10(7):813-22.

6. Kokich JR, Kiyak HA, Shapiro PA. Comparing the perception of dentists and lay people to altered dental esthetics. J Esthet Dent 1999;11(6):311-24.

7. Hunt O, Johnston C, Hepper P, Burden D, Hepper P, Stevenson, M. The influence of maxillary gingival exposure on dental attractiveness ratings. Eur J Orthod. 2002;24(2):199-204.

8. Geron S, Atalia W. Influence of sex on the perception of oral and smile esthetics with differente gingival display and incisal plane inclination. Angle Orthod 2005;75(5):778-84.

9. Roden-Johnson D, Gallerano R, English J. The effects of buccal corridor spaces and arch form on smile esthetics. Am J OrthodDentofacialOrthop. 2005;127(3):343-50.

10.Pinho S, Ciriaco C, Faber J, Lenza MA. Impact of dental asymmetries on the perception of smile esthetics. Am J
OrthodDentofacialOrthop. 2007;132(6):748-53.

11.Scott CR, Goonewardene MS, Murray K. Influence of lips on the perception of malocclusion. Am J OrthodDentofacialOrthop. 2006;130(2):152-62.

12.Wikipedia, the free enciclopedia.htm. Pixels per inch. $2005 \mathrm{http}: / /$ en.wikipedia.org/wiki/Pixels_per_inch.

13.Ohnhaus EE, Adler R. Methodological problems in the measurement of pain: a comparison between the verbal rating scale and the visual analogue scale. Pain. 1975;1(4):379-84.

14.Ritter DE, Gandini LG, Pinto Ados S, Locks A. Esthetic influence of negative space in the buccal corridor during smiling. Angle Orthod. 2006;76(2):198-203.

15.Roden-Johnson D, Gallerano R, English J. The effects of buccal corridors spaces and arch form on smile esthetics. Am J OrthodDentofacialOrthop. 2005;127(3):343-50.

16.Martin AJ, Buschang PH, Boley JC, Taylor RW, McKinney TW. The impact of buccal corridors on smile attractiveness. Eur J Orthod. 2007;29(5):530-7.

17.Krishnan V, Daniel ST, Lazar D, Asok A. Characterization of posed smile by using visual analog scale, smile arc, buccal corridor measures, and modified smile index. Am J OrthodDentofacialOrthop. 2008;133(4):515-23.

18.Parekh SM, Fields HW, Beck M, Rosenstiel S. Attractiveness of variations in the smile arc and buccal corridor spaces as judged by orthodontists and laymen. Angle Orthod. 2006;76(4):557-63.

19.Ioi H, Nakata S, Counts AL. Effects of buccal corridors on smile esthetics in Japanese. Angle Orthod. 2009;79(4):628-33.

20.Roden-Johnson D, Gallerano R, English J. The effects of buccal corridor spaces and arch form on smile esthetics. Am J Orthod Dentofacial Orthop. 2005;127(3):343-50.

21.Pinho S, Ciriaco C, Faber J, Lenza MA. Impact of dental asymmetries on the perception of smile esthetics. Am J Orthod Dentofacial Orthop. 2007;132(6):748-53.

22.Scott CR, Goonewardene MS, Murray K. Influence of lips on the perception of malocclusion. Am J Orthod Dentofacial Orthop. 2006;130(2):152-62.

23.Ioi H, Nakata $\mathrm{S}$, Counts AL. Influence of gingival display on smile aesthetics in Japanese. Eur J Orthod. 2010;32(6):633-7.

24.Abu Alhaija ES, Al-Shamsi NO, Al-Khateeb S. Perceptions of Jordanian laypersons and dental professionals to altered smile aesthetics. Eur J Orthod. 2011;33(4):450-6.

25.Moore T, Southard KA, Casko JS, Qian F, Southard TE. Buccal corridor and smile esthetics. Am J OrthodDentofacialOrthop. 2005;127(2):208-13.

26.Sarver DM. Principles of cosmetic dentistry in orthodontics: Shape and proportionality of 
anterior teeth. Am J OrthodDentofacialOrthop. 2004;126(6):749- 53.

27.Margolis MJ. Esthetic considerations in orthodontic treatment of adults. Dent Clin North Am. 1997;41(1):2948.
28.Peck S, Peck L, Kataja M. The gingival smile line. AngleOrthod. 1992;62(2):91-100.

29.Durgekar SG, K N, Naik V.The ideal smile and its orthodontic implications. World J Orthod. 2010;11(3):211-20.

Recibido : 05-02-2012

Aceptado: 28-06-2012 